\title{
Equivalent crossed products and cross product bialgebras *
}

\author{
Florin Panaite \\ Institute of Mathematics of the Romanian Academy \\ PO-Box 1-764, RO-014700 Bucharest, Romania \\ e-mail: Florin.Panaite@imar.ro \\ Dedicated to Fred Van Oystaeyen, on the occasion of his $65^{\text {th }}$ birthday
}

\begin{abstract}
In a previous paper we proved a result of the type "invariance under twisting" for Brzeziński's crossed products. In this paper we prove a converse of this result, obtaining thus a characterization of what we call equivalent crossed products. As an application, we characterize cross product bialgebras (in the sense of Bespalov and Drabant) that are equivalent (in a certain sense) to a given cross product bialgebra in which one of the factors is a bialgebra and whose coalgebra structure is a tensor product coalgebra.
\end{abstract}

\section{Introduction}

In [4], Brzeziński introduced a very general construction, called crossed product, containing as particular cases several important constructions introduced before, such as twisted tensor products of algebras (as in [6], [12]) and classical Hopf crossed products. Given an (associative unital) algebra $A$, a vector space $V$ endowed with a distinguished element $1_{V}$ and two linear maps $\sigma: V \otimes V \rightarrow A \otimes V$ and $R: V \otimes A \rightarrow A \otimes V$ satisfying certain conditions, Brzeziński's crossed product is a certain (associative unital) algebra structure on $A \otimes V$, denoted in what follows by $A \otimes_{R, \sigma} V$.

In [10] we proved a result of the type invariance under twisting for crossed products (containing as particular cases the invariance under twisting for twisted tensor products of algebras from [8] and the invariance under twisting for quasi-Hopf smash products from [5]) as follows: if $A \otimes_{R, \sigma} V$ is a crossed product and $\theta, \gamma: V \rightarrow A \otimes V$ are linear maps, we can define certain maps $\sigma^{\prime}: V \otimes V \rightarrow A \otimes V$ and $R^{\prime}: V \otimes A \rightarrow A \otimes V$ and if some conditions are satisfied then $A \otimes_{R^{\prime}, \sigma^{\prime}} V$ is a crossed product, isomorphic to $A \otimes_{R, \sigma} V$.

Our first aim here is to prove a converse of this result. First, we call two crossed products $A \otimes_{R, \sigma} V$ and $A \otimes_{R^{\prime}, \sigma^{\prime}} V$ equivalent if there exists a linear isomorphism $\varphi: A \otimes_{R^{\prime}, \sigma^{\prime}} V \simeq A \otimes_{R, \sigma} V$ that is an algebra map and a morphism of left $A$-modules. With this terminology, our result says that two crossed products $A \otimes_{R, \sigma} V$ and $A \otimes_{R^{\prime}, \sigma^{\prime}} V$ are equivalent if and only if there exist linear maps $\theta, \gamma: V \rightarrow A \otimes V$ satisfying a certain list of conditions.

There exists a dual construction to Brzeziński's crossed product, called crossed coproduct. A cross product bialgebra, as defined by Bespalov and Drabant in [3, is a bialgebra whose algebra structure is a crossed product algebra and whose coalgebra structure is a crossed coproduct

${ }^{*}$ This work was supported by a grant of the Romanian National Authority for Scientific Research, CNCSUEFISCDI, project number PN-II-ID-PCE-2011-3-0635, contract nr. 253/5.10.2011. 
coalgebra. If $A_{W}^{\rho} \bowtie_{R}^{\sigma} C$ and $A_{W^{\prime}}^{\rho^{\prime}} \bowtie_{R^{\prime}}^{\sigma^{\prime}} C$ are cross product bialgebras, we call them equivalent if there exists a linear isomorphism $\varphi: A_{W^{\prime}}^{\rho^{\prime}} \bowtie_{R^{\prime}}^{\sigma^{\prime}} C \simeq A_{W}^{\rho} \bowtie_{R}^{\sigma} C$ that is a morphism of bialgebras, of left $A$-modules and of right $C$-comodules. It is a natural problem to characterize all cross product bialgebras $A_{W^{\prime}}^{\rho^{\prime}} \bowtie_{R^{\prime}}^{\sigma^{\prime}} C$ that are equivalent to a given cross product bialgebra $A_{W}^{\rho} \bowtie_{R}^{\sigma} C$. We can solve here only a particular case of this problem, namely the case in which we assume that $A$ is a bialgebra and the coalgebra structure of the given cross product bialgebra is the tensor product coalgebra.

\section{Preliminaries}

We work over a commutative field $k$. All algebras, linear spaces etc. will be over $k$; unadorned $\otimes$ means $\otimes_{k}$. By "algebra" (respectively "coalgebra") we always mean an associative unital algebra (respectively coassociative counital coalgebra). For the comultiplication of a coalgebra $C$, we use the version of Sweedler's sigma notation $\Delta(c)=c_{1} \otimes c_{2}$ for all $c \in C$.

We recall from [6], [12] that, given two algebras $A, B$ and a $k$-linear map $R: B \otimes A \rightarrow A \otimes B$, with notation $R(b \otimes a)=a_{R} \otimes b_{R}$, for $a \in A, b \in B$, satisfying the conditions $a_{R} \otimes 1_{R}=a \otimes 1$, $1_{R} \otimes b_{R}=1 \otimes b,\left(a a^{\prime}\right)_{R} \otimes b_{R}=a_{R} a_{r}^{\prime} \otimes b_{R_{r}}, a_{R} \otimes\left(b b^{\prime}\right)_{R}=a_{R_{r}} \otimes b_{r} b_{R}^{\prime}$, for all $a, a^{\prime} \in A$ and $b, b^{\prime} \in B$ (where $r$ and $R$ are two different indices), if we define on $A \otimes B$ a new multiplication, by $(a \otimes b)\left(a^{\prime} \otimes b^{\prime}\right)=a a_{R}^{\prime} \otimes b_{R} b^{\prime}$, then this multiplication is associative with unit $1 \otimes 1$. In this case, the map $R$ is called a twisting map between $A$ and $B$ and the new algebra structure on $A \otimes B$ is denoted by $A \otimes_{R} B$ and called the twisted tensor product of $A$ and $B$ afforded by $R$.

We recall from [4] the construction of Brzeziński's crossed product:

Proposition 1.1 ([4]) Let $\left(A, \mu, 1_{A}\right)$ be an (associative unital) algebra and $V$ a vector space equipped with a distinguished element $1_{V} \in V$. Then the vector space $A \otimes V$ is an associative algebra with unit $1_{A} \otimes 1_{V}$ and whose multiplication has the property that $\left(a \otimes 1_{V}\right)(b \otimes v)=a b \otimes v$, for all $a, b \in A$ and $v \in V$, if and only if there exist linear maps $\sigma: V \otimes V \rightarrow A \otimes V$ and $R: V \otimes A \rightarrow A \otimes V$ satisfying the following conditions:

$$
\begin{aligned}
& R\left(1_{V} \otimes a\right)=a \otimes 1_{V}, \quad R\left(v \otimes 1_{A}\right)=1_{A} \otimes v, \quad \forall a \in A, v \in V, \\
& \sigma\left(1_{V}, v\right)=\sigma\left(v, 1_{V}\right)=1_{A} \otimes v, \quad \forall v \in V, \\
& R \circ\left(i d_{V} \otimes \mu\right)=\left(\mu \otimes i d_{V}\right) \circ\left(i d_{A} \otimes R\right) \circ\left(R \otimes i d_{A}\right), \\
& \left(\mu \otimes i d_{V}\right) \circ\left(i d_{A} \otimes \sigma\right) \circ\left(R \otimes i d_{V}\right) \circ\left(i d_{V} \otimes \sigma\right) \\
& \quad=\left(\mu \otimes i d_{V}\right) \circ\left(i d_{A} \otimes \sigma\right) \circ\left(\sigma \otimes i d_{V}\right), \\
& \left(\mu \otimes i d_{V}\right) \circ\left(i d_{A} \otimes \sigma\right) \circ\left(R \otimes i d_{V}\right) \circ\left(i d_{V} \otimes R\right) \\
& \quad=\left(\mu \otimes i d_{V}\right) \circ\left(i d_{A} \otimes R\right) \circ\left(\sigma \otimes i d_{A}\right) .
\end{aligned}
$$

If this is the case, the multiplication of $A \otimes V$ is given explicitly by

$$
\mu_{A \otimes V}=\left(\mu_{2} \otimes i d_{V}\right) \circ\left(i d_{A} \otimes i d_{A} \otimes \sigma\right) \circ\left(i d_{A} \otimes R \otimes i d_{V}\right)
$$

where $\mu_{2}=\mu \circ\left(i d_{A} \otimes \mu\right)=\mu \circ\left(\mu \otimes i d_{A}\right)$. We denote by $A \otimes_{R, \sigma} V$ this algebra structure and call it the crossed product afforded by the data $(A, V, R, \sigma)$.

If $A \otimes_{R, \sigma} V$ is a crossed product, we introduce the following Sweedler-type notation:

$$
R: V \otimes A \rightarrow A \otimes V, \quad R(v \otimes a)=a_{R} \otimes v_{R},
$$




$$
\sigma: V \otimes V \rightarrow A \otimes V, \quad \sigma\left(v, v^{\prime}\right)=\sigma_{1}\left(v, v^{\prime}\right) \otimes \sigma_{2}\left(v, v^{\prime}\right)
$$

for all $v, v^{\prime} \in V$ and $a \in A$. With this notation, the multiplication of $A \otimes_{R, \sigma} V$ reads

$$
(a \otimes v)\left(a^{\prime} \otimes v^{\prime}\right)=a a_{R}^{\prime} \sigma_{1}\left(v_{R}, v^{\prime}\right) \otimes \sigma_{2}\left(v_{R}, v^{\prime}\right), \quad \forall a, a^{\prime} \in A, v, v^{\prime} \in V .
$$

A twisted tensor product is a particular case of a crossed product (cf. [7]), namely, if $A \otimes_{R} B$ is a twisted tensor product of algebras then $A \otimes_{R} B=A \otimes_{R, \sigma} B$, where $\sigma: B \otimes B \rightarrow A \otimes B$ is given by $\sigma\left(b, b^{\prime}\right)=1_{A} \otimes b b^{\prime}$, for all $b, b^{\prime} \in B$.

We recall from [10] the invariance under twisting for crossed products:

Theorem 1.2 Let $A \otimes_{R, \sigma} V$ be a crossed product and assume we are given two linear maps $\theta, \gamma: V \rightarrow A \otimes V$, with notation $\theta(v)=v_{<-1>} \otimes v_{<0>}$ and $\gamma(v)=v_{\{-1\}} \otimes v_{\{0\}}$, for all $v \in V$. Define the maps $R^{\prime}: V \otimes A \rightarrow A \otimes V$ and $\sigma^{\prime}: V \otimes V \rightarrow A \otimes V$ by the formulae

$$
\begin{aligned}
& R^{\prime}=\left(\mu_{2} \otimes i d_{V}\right) \circ\left(i d_{A} \otimes i d_{A} \otimes \gamma\right) \circ\left(i d_{A} \otimes R\right) \circ\left(\theta \otimes i d_{A}\right), \\
& \sigma^{\prime}=\left(\mu \otimes i d_{V}\right) \circ\left(i d_{A} \otimes \gamma\right) \circ\left(\mu_{2} \otimes i d_{V}\right) \circ\left(i d_{A} \otimes i d_{A} \otimes \sigma\right) \\
& \circ\left(i d_{A} \otimes R \otimes i d_{V}\right) \circ(\theta \otimes \theta) .
\end{aligned}
$$

Assume that the following conditions are satisfied:

$$
\begin{aligned}
& \theta\left(1_{V}\right)=1_{A} \otimes 1_{V}, \quad \gamma\left(1_{V}\right)=1_{A} \otimes 1_{V}, \\
& v_{<-1>} v_{<0>_{\{-1\}}} \otimes v_{<0>_{\{0\}}}=1_{A} \otimes v, \quad \forall v \in V, \\
& v_{\{-1\}} v_{\{0\}_{<-1>}} \otimes v_{\{0\}_{<0>}}=1_{A} \otimes v, \quad \forall v \in V, \\
& \left(\mu \otimes i d_{V}\right) \circ\left(\mu \otimes \sigma^{\prime}\right) \circ\left(i d_{A} \otimes \gamma \otimes i d_{V}\right) \circ\left(R \otimes i d_{V}\right) \circ\left(i d_{V} \otimes \gamma\right) \\
& \quad=\left(\mu \otimes i d_{V}\right) \circ\left(i d_{A} \otimes \gamma\right) \circ \sigma .
\end{aligned}
$$

Then $A \otimes_{R^{\prime}, \sigma^{\prime}} V$ is a crossed product and we have an algebra isomorphism $\varphi: A \otimes_{R^{\prime}, \sigma^{\prime}} V \simeq$ $A \otimes_{R, \sigma} V, \varphi(a \otimes v)=a v_{<-1>} \otimes v_{<0>}$, for all $a \in A, v \in V$.

\section{The characterization of equivalent crossed products}

Definition 2.1 Let $\left(A, \mu, 1_{A}\right)$ be an (associative unital) algebra, $V$ a vector space equipped with a distinguished element $1_{V} \in V$ and $A \otimes_{R, \sigma} V, A \otimes_{R^{\prime}, \sigma^{\prime}} V$ two crossed products. We say that $A \otimes_{R, \sigma} V$ and $A \otimes_{R^{\prime}, \sigma^{\prime}} V$ are equivalent if there exists an algebra isomorphism $\varphi: A \otimes_{R^{\prime}, \sigma^{\prime}} V \simeq$ $A \otimes_{R, \sigma} V$ with the property that $\varphi\left(a \otimes 1_{V}\right)=a \otimes 1_{V}$ for all $a \in A$.

Remark 2.2 A crossed product algebra $A \otimes_{R, \sigma} V$ becomes canonically a left $A$-module, by

$$
A \otimes\left(A \otimes_{R, \sigma} V\right) \rightarrow A \otimes_{R, \sigma} V, \quad a^{\prime} \otimes(a \otimes v) \mapsto a^{\prime} a \otimes v .
$$

Then it is easy to see that two crossed products $A \otimes_{R, \sigma} V$ and $A \otimes_{R^{\prime}, \sigma^{\prime}} V$ are equivalent if and only if there exists a linear isomorphism $\varphi: A \otimes_{R^{\prime}, \sigma^{\prime}} V \simeq A \otimes_{R, \sigma} V$ which is an algebra map and a morphism of left A-modules.

Theorem 2.3 Let $A \otimes_{R, \sigma} V$ and $A \otimes_{R^{\prime}, \sigma^{\prime}} V$ be two crossed products. Then $A \otimes_{R, \sigma} V$ and $A \otimes_{R^{\prime}, \sigma^{\prime}} V$ are equivalent if and only if there exist linear maps $\theta, \gamma: V \rightarrow A \otimes V$, with notation $\theta(v)=v_{<-1>} \otimes v_{<0>}$ and $\gamma(v)=v_{\{-1\}} \otimes v_{\{0\}}$, for all $v \in V$, such that the conditions (1.6)-(1.11) are satisfied. 
Proof. One implication is exactly Theorem 1.2, so we only have to prove the converse. Assume that there exists an algebra isomorphism $\varphi: A \otimes_{R^{\prime}, \sigma^{\prime}} V \simeq A \otimes_{R, \sigma} V$ such that $\varphi\left(a \otimes 1_{V}\right)=a \otimes 1_{V}$ for all $a \in A$, with inverse denoted by $\varphi^{-1}: A \otimes_{R, \sigma} V \simeq A \otimes_{R^{\prime}, \sigma^{\prime}} V$. Define the maps

$$
\begin{aligned}
& \theta: V \rightarrow A \otimes V, \quad \theta(v)=\varphi\left(1_{A} \otimes v\right):=v_{<-1>} \otimes v_{<0>}, \\
& \gamma: V \rightarrow A \otimes V, \quad \gamma(v)=\varphi^{-1}\left(1_{A} \otimes v\right):=v_{\{-1\}} \otimes v_{\{0\}} .
\end{aligned}
$$

Since $\varphi$ is an algebra isomorphism, for all $a \in A, v \in V$ we have $\varphi(a \otimes v)=\varphi\left(\left(a \otimes 1_{V}\right)\left(1_{A} \otimes v\right)\right)=$ $\varphi\left(a \otimes 1_{V}\right) \varphi\left(1_{A} \otimes v\right)=\left(a \otimes 1_{V}\right)\left(v_{<-1>} \otimes v_{<0>}\right)=a v_{<-1>} \otimes v_{<0>}$ and similarly $\varphi^{-1}(a \otimes v)=$ $a v_{\{-1\}} \otimes v_{\{0\}}$. Since $\varphi\left(1_{A} \otimes 1_{V}\right)=\varphi^{-1}\left(1_{A} \otimes 1_{V}\right)=1_{A} \otimes 1_{V}$ we obtain $\theta\left(1_{V}\right)=\gamma\left(1_{V}\right)=1_{A} \otimes 1_{V}$, that is (1.8) holds. Now we compute, for $v \in V$ :

$$
\begin{aligned}
& 1_{A} \otimes v=\varphi^{-1}\left(\varphi\left(1_{A} \otimes v\right)\right)=\varphi^{-1}\left(v_{<-1>} \otimes v_{<0>}\right)=v_{<-1>} v_{<0>\{-1\}} \otimes v_{<0>\{0\}}, \\
& 1_{A} \otimes v=\varphi\left(\varphi^{-1}\left(1_{A} \otimes v\right)\right)=\varphi\left(v_{\{-1\}} \otimes v_{\{0\}}\right)=v_{\{-1\}} v_{\{0\}<-1>} \otimes v_{\{0\}<0>},
\end{aligned}
$$

that is (1.9) and (1.10) hold. Let now $v \in V, a \in A$; we compute:

$$
\begin{aligned}
& R^{\prime}(v \otimes a)=\varphi^{-1}\left(\varphi\left(R^{\prime}(v \otimes a)\right)\right) \\
& =\varphi^{-1}\left(\varphi\left(\left(1_{A} \otimes v\right)\left(a \otimes 1_{V}\right)\right)\right) \\
& =\varphi^{-1}\left(\varphi\left(1_{A} \otimes v\right) \varphi\left(a \otimes 1_{V}\right)\right) \\
& =\varphi^{-1}\left(\left(v_{<-1>} \otimes v_{<0>}\right)\left(a \otimes 1_{V}\right)\right) \\
& =\varphi^{-1}\left(v_{<-1>} a_{R} \otimes v_{<0>R}\right) \\
& =v_{<-1>} a_{R} v_{<0>_{R_{\{-1\}}}} \otimes v_{<0>_{R_{\{0\}}}},
\end{aligned}
$$

and this is exactly the relation (1.6). Let now $v, w \in V$; we compute:

$$
\begin{aligned}
\sigma^{\prime}(v, w) & =\varphi^{-1}\left(\varphi\left(\sigma^{\prime}(v, w)\right)\right) \\
& =\varphi^{-1}\left(\varphi\left(\left(1_{A} \otimes v\right)\left(1_{A} \otimes w\right)\right)\right) \\
& =\varphi^{-1}\left(\varphi\left(1_{A} \otimes v\right) \varphi\left(1_{A} \otimes w\right)\right) \\
& =\varphi^{-1}\left(\left(v_{<-1>} \otimes v_{<0>}\right)\left(w_{<-1>} \otimes w_{<0>}\right)\right) \\
& =\varphi^{-1}\left(v_{<-1>} w_{<-1>_{R}} \sigma_{1}\left(v_{<0>_{R}}, w_{<0>}\right) \otimes \sigma_{2}\left(v_{<0>_{R}}, w_{<0>}\right)\right) \\
& =v_{<-1>} w_{<-1>_{R}} \sigma_{1}\left(v_{<0>_{R}}, w_{<0>}\right) \sigma_{2}\left(v_{<0>_{R}}, w_{<0>}\right)_{\{-1\}} \otimes \sigma_{2}\left(v_{<0>_{R}}, w_{<0>}\right)_{\{0\}},
\end{aligned}
$$

and this is exactly the relation (1.7).

The only thing left to prove is the relation (1.11). In order to show that the left hand side and the right hand side in (1.11) are equal, it is enough to prove that they are equal after composing with $\varphi$. Thus, for $v, w \in V$, we compute (denoting by $r$ another copy of $R$ ):

$$
\begin{aligned}
& \varphi \circ\left(\mu \otimes i d_{V}\right) \circ\left(\mu \otimes \sigma^{\prime}\right) \circ\left(i d_{A} \otimes \gamma \otimes i d_{V}\right) \circ\left(R \otimes i d_{V}\right) \circ\left(i d_{V} \otimes \gamma\right)(v \otimes w) \\
& =\varphi \circ\left(\mu \otimes i d_{V}\right) \circ\left(\mu \otimes \sigma^{\prime}\right) \circ\left(i d_{A} \otimes \gamma \otimes i d_{V}\right) \circ\left(R \otimes i d_{V}\right)\left(v \otimes w_{\{-1\}} \otimes w_{\{0\}}\right) \\
& =\varphi \circ\left(\mu \otimes i d_{V}\right) \circ\left(\mu \otimes \sigma^{\prime}\right) \circ\left(i d_{A} \otimes \gamma \otimes i d_{V}\right)\left(w_{\{-1\}_{R}} \otimes v_{R} \otimes w_{\{0\}}\right) \\
& =\varphi \circ\left(\mu \otimes i d_{V}\right) \circ\left(\mu \otimes \sigma^{\prime}\right)\left(w_{\{-1\}_{R}} \otimes v_{R_{\{-1\}}} \otimes v_{R_{\{0\}}} \otimes w_{\{0\}}\right) \\
& \stackrel{1.7}{=} \varphi \circ\left(\mu \otimes i d_{V}\right)\left(w_{\{-1\}_{R}} v_{R_{\{-1\}}} \otimes v_{R_{\{0\}<-1>}} w_{\{0\}_{<-1>r}} \sigma_{1}\left(v_{R_{\{0\}<0>r}}, w_{\{0\}_{<0>}}\right)\right. \\
& \left.\sigma_{2}\left(v_{R_{\{0\}<0>r}}, w_{\{0\}<0>}\right)_{\{-1\}} \otimes \sigma_{2}\left(v_{R_{\{0\}<0>r}}, w_{\{0\}<0>}\right)_{\{0\}}\right)
\end{aligned}
$$




$$
\begin{aligned}
& =\varphi\left(w_{\{-1\}_{R}} v_{R_{\{-1\}}} v_{R_{\{0\}<-1>}} w_{\{0\}_{<-1>r}} \sigma_{1}\left(v_{R_{\{0\}<0>r}}, w_{\{0\}<0>}\right)\right. \\
& \left.\sigma_{2}\left(v_{R_{\{0\}<0>r}}, w_{\{0\}_{<0>}}\right)_{\{-1\}} \otimes \sigma_{2}\left(v_{R_{\{0\}<0>r}}, w_{\{0\}<0>}\right)_{\{0\}}\right) \\
& =w_{\{-1\}_{R}} v_{R_{\{-1\}}} v_{R_{\{0\}<-1>}} w_{\{0\}_{<-1>r}} \sigma_{1}\left(v_{R_{\{0\}<0>r}}, w_{\{0\}<0>}\right) \\
& \sigma_{2}\left(v_{R_{\{0\}<0>r}}, w_{\{0\}_{<0>}}\right)_{\{-1\}} \sigma_{2}\left(v_{R_{\{0\}<0>r}}, w_{\{0\}<0>}\right)_{\{0\}<-1>} \\
& \otimes \sigma_{2}\left(v_{R_{\{0\}<0>r}}, w_{\{0\}<0>}\right)\{0\}<0> \\
& \stackrel{1.10}{=} w_{\{-1\}_{R}} v_{R_{\{-1\}}} v_{R_{\{0\}<-1>}} w_{\{0\}_{<-1>r}} \sigma_{1}\left(v_{R_{\{0\}<0>r}}, w_{\{0\}<0>}\right) \\
& \otimes \sigma_{2}\left(v_{R_{\{0\}<0>r}}, w_{\{0\}<0>}\right) \\
& \text { 1.10) } w_{\{-1\}_{R}} w_{\{0\}_{<-1>r}} \sigma_{1}\left(v_{R_{r}}, w_{\{0\}<0>}\right) \otimes \sigma_{2}\left(v_{R_{r}}, w_{\{0\}<0>}\right) \\
& \stackrel{1.3}{=}\left(w_{\{-1\}} w_{\{0\}_{<-1>}}\right)_{R} \sigma_{1}\left(v_{R}, w_{\{0\}_{<0>}}\right) \otimes \sigma_{2}\left(v_{R}, w_{\{0\}_{<0>}}\right) \\
& \stackrel{1.10}{=}\left(1_{A}\right)_{R} \sigma_{1}\left(v_{R}, w\right) \otimes \sigma_{2}\left(v_{R}, w\right) \\
& \stackrel{1.1}{=} \sigma(v \otimes w) \text {, } \\
& \varphi \circ\left(\mu \otimes i d_{V}\right) \circ\left(i d_{A} \otimes \gamma\right) \circ \sigma(v \otimes w) \\
& =\varphi \circ\left(\mu \otimes i d_{V}\right) \circ\left(i d_{A} \otimes \gamma\right)\left(\sigma_{1}(v, w) \otimes \sigma_{2}(v, w)\right) \\
& =\varphi \circ\left(\mu \otimes i d_{V}\right)\left(\sigma_{1}(v, w) \otimes \sigma_{2}(v, w)_{\{-1\}} \otimes \sigma_{2}(v, w)_{\{0\}}\right) \\
& =\varphi\left(\sigma_{1}(v, w) \sigma_{2}(v, w)_{\{-1\}} \otimes \sigma_{2}(v, w)_{\{0\}}\right) \\
& =\sigma_{1}(v, w) \sigma_{2}(v, w)_{\{-1\}} \sigma_{2}(v, w)_{\{0\}<-1>} \otimes \sigma_{2}(v, w)_{\{0\}<0>} \\
& \stackrel{1.10}{=} \sigma_{1}(v, w) \otimes \sigma_{2}(v, w) \\
& =\sigma(v \otimes w) \text {, }
\end{aligned}
$$

and we can see that the two terms are equal.

With a similar proof, we can obtain a converse of the invariance under twisting for twisted tensor products of algebras from [8]:

Definition 2.4 Let $A$ be an associative unital algebra and $B, B^{\prime}$ two associative unital algebra structures with the same unit $1_{B}$ on the vector space $B$. If $A \otimes_{R} B$ and $A \otimes_{R^{\prime}} B^{\prime}$ are two twisted tensor products of algebras, we call them A-equivalent if there exists an algebra isomorphism $\varphi: A \otimes_{R^{\prime}} B^{\prime} \simeq A \otimes_{R} B$ such that $\varphi\left(a \otimes 1_{B}\right)=a \otimes 1_{B}$ for all $a \in A$.

Theorem 2.5 Let $A$ be an associative unital algebra and $B, B^{\prime}$ two associative unital algebra structures with the same unit $1_{B}$ on the vector space $B$. Denote the multiplication of $B$ by $b \otimes b^{\prime} \mapsto b b^{\prime}$ and the multiplication of $B^{\prime}$ by $b \otimes b^{\prime} \mapsto b * b^{\prime}$. If $A \otimes_{R} B$ and $A \otimes_{R^{\prime}} B^{\prime}$ are two twisted tensor products of algebras, then they are $A$-equivalent if and only if there exist two linear maps $\theta, \gamma: B \rightarrow A \otimes B$, with notation $\theta(b)=b_{<-1>} \otimes b_{<0>}$ and $\gamma(b)=b_{\{-1\}} \otimes b_{\{0\}}$, such that $\theta$ is an algebra map from $B^{\prime}$ to $A \otimes_{R} B, \gamma\left(1_{B}\right)=1_{A} \otimes 1_{B}$ and for all $a \in A$ and $b, b^{\prime} \in B$ we have:

$$
\begin{aligned}
& \gamma\left(b b^{\prime}\right)=b_{\{-1\}_{R}}^{\prime} b_{R_{\{-1\}}} \otimes b_{R_{\{0\}}} * b_{\{0\}}^{\prime}, \\
& b_{<-1>} b_{<0>\{-1\}} \otimes b_{<0>\{0\}}=1 \otimes b, \\
& b_{\{-1\}} b_{\{0\}_{<-1>}} \otimes b_{\{0\}_{<0>}}=1 \otimes b, \\
& R^{\prime}(b \otimes a)=b_{<-1>} a_{R} b_{<0>_{R_{\{-1\}}}} \otimes b_{<0>_{R_{\{0\}}}} .
\end{aligned}
$$


Remark 2.6 The same type of result as in Theorem [2.3] was proved in [2] for bicrossed products of Hopf algebras.

\section{Equivalent cross product bialgebras}

We begin by recalling the dual concept of Brzeziński's crossed products, which appears (in a slightly different form) in [3]:

Proposition 3.1 Let $\left(C, \Delta_{C}, \varepsilon_{C}\right)$ be a (coassociative counital) coalgebra and $X$ a vector space endowed with a linear map $\varepsilon_{X}: X \rightarrow k$. Then the vector space $X \otimes C$ is a coassociative coalgebra with counit $\varepsilon_{X} \otimes \varepsilon_{C}$ and whose comultiplication $\Delta$ has the property that $\left(i d_{X} \otimes i d_{C} \otimes \varepsilon_{X} \otimes i d_{C}\right) \circ \Delta=$ $i d_{X} \otimes \Delta_{C}$ if and only if there exist linear maps $W: X \otimes C \rightarrow C \otimes X$ and $\rho: X \otimes C \rightarrow X \otimes X$ satisfying the following conditions:

$$
\begin{aligned}
& \left(i d_{C} \otimes \varepsilon_{X}\right) \circ W=\varepsilon_{X} \otimes i d_{C}, \quad\left(\varepsilon_{C} \otimes i d_{X}\right) \circ W=i d_{X} \otimes \varepsilon_{C}, \\
& \left(i d_{X} \otimes \varepsilon_{X}\right) \circ \rho=i d_{X} \otimes \varepsilon_{C}, \quad\left(\varepsilon_{X} \otimes i d_{X}\right) \circ \rho=i d_{X} \otimes \varepsilon_{C}, \\
& \left(\Delta_{C} \otimes i d_{X}\right) \circ W=\left(i d_{C} \otimes W\right) \circ\left(W \otimes i d_{C}\right) \circ\left(i d_{X} \otimes \Delta_{C}\right), \\
& \left(\rho \otimes i d_{X}\right) \circ\left(i d_{X} \otimes W\right) \circ\left(\rho \otimes i d_{C}\right) \circ\left(i d_{X} \otimes \Delta_{C}\right) \\
& \quad=\left(i d_{X} \otimes \rho\right) \circ\left(\rho \otimes i d_{C}\right) \circ\left(i d_{X} \otimes \Delta_{C}\right), \\
& \left(W \otimes i d_{X}\right) \circ\left(i d_{X} \otimes W\right) \circ\left(\rho \otimes i d_{C}\right) \circ\left(i d_{X} \otimes \Delta_{C}\right) \\
& \quad=\left(i d_{C} \otimes \rho\right) \circ\left(W \otimes i d_{C}\right) \circ\left(i d_{X} \otimes \Delta_{C}\right) .
\end{aligned}
$$

In this case, the comultiplication of $X \otimes C$ is given explicitely by

$$
\Delta=\left(i d_{X} \otimes W \otimes i d_{C}\right) \circ\left(\rho \otimes \Delta_{C}\right) \circ\left(i d_{X} \otimes \Delta_{C}\right) .
$$

We denote by $X_{W, \rho} \otimes C$ this coalgebra structure and call it the crossed coproduct afforded by the data $(X, C, W, \rho)$.

If $X_{W, \rho} \otimes C$ is a crossed coproduct, we introduce the following Sweedler-type notation:

$$
\begin{aligned}
& W: X \otimes C \rightarrow C \otimes X, \quad W(x \otimes c)=c_{W} \otimes x_{W}, \\
& \rho: X \otimes C \rightarrow X \otimes X, \quad \rho(x \otimes c)=\rho_{1}(x, c) \otimes \rho_{2}(x, c),
\end{aligned}
$$

for all $x \in X, c \in C$. With this notation, the comultiplication of $X_{W, \rho} \otimes C$ reads:

$$
\Delta(x \otimes c)=\left(\rho_{1}\left(x, c_{1}\right) \otimes c_{2_{W}}\right) \otimes\left(\rho_{2}\left(x, c_{1}\right)_{W} \otimes c_{3}\right), \quad \forall x \in X, c \in C .
$$

Remark 3.2 It is easy to see that, if $X_{W, \rho} \otimes C$ is a crossed coproduct as above, then

$$
\begin{aligned}
& W=\left(\varepsilon_{X} \otimes i d_{C} \otimes i d_{X} \otimes \varepsilon_{C}\right) \circ \Delta, \\
& \rho=\left(i d_{X} \otimes \varepsilon_{C} \otimes i d_{X} \otimes \varepsilon_{C}\right) \circ \Delta,
\end{aligned}
$$

where $\Delta$ is the comultiplication of $X_{W, \rho} \otimes C$.

We recall the following concept from [3]: 
Definition 3.3 ([3]) A bialgebra $B$ is called a cross product bialgebra if its underlying algebra structure is a crossed product algebra $A \otimes_{R, \sigma} C$ and its underlying coalgebra structure is a crossed coproduct coalgebra $A_{W, \rho} \otimes C$ on the same objects. This cross product bialgebra $B$ will be denoted by $A_{W}^{\rho} \bowtie_{R}^{\sigma} C$.

Remark 3.4 If $A_{W}^{\rho} \bowtie_{R}^{\sigma} C$ is a cross product bialgebra, then the vector space $A \otimes C$ becomes canonically a left $A$-module and a right $C$-comodule as follows:

$$
\begin{aligned}
& A \otimes(A \otimes C) \rightarrow A \otimes C, \quad a^{\prime} \otimes(a \otimes c) \mapsto a^{\prime} a \otimes c, \\
& A \otimes C \rightarrow(A \otimes C) \otimes C, \quad a \otimes c \mapsto\left(a \otimes c_{1}\right) \otimes c_{2},
\end{aligned}
$$

for all $a, a^{\prime} \in A$ and $c \in C$.

Definition 3.5 Two cross product bialgebras $A_{W}^{\rho} \bowtie_{R}^{\sigma} C$ and $A_{W^{\prime}}^{\rho^{\prime}} \bowtie_{R^{\prime}}^{\sigma^{\prime}} C$ will be called equivalent if there exists a linear isomorphism $\varphi: A_{W^{\prime}}^{\rho^{\prime}} \bowtie_{R^{\prime}}^{\sigma^{\prime}} C \simeq A_{W}^{\rho} \bowtie_{R}^{\sigma} C$ which is a morphism of bialgebras, of left $A$-modules and of right $C$-comodules.

Let now $\left(A, \mu_{A}, 1_{A}, \Delta_{A}, \varepsilon_{A}\right)$ be a bialgebra and $\left(C, \Delta_{C}, \varepsilon_{C}\right)$ a coalgebra. Assume that we have a crossed product algebra $A \otimes_{R, \sigma} C$. Define the maps

$$
\begin{aligned}
& W_{0}: A \otimes C \rightarrow C \otimes A, \quad W_{0}(a \otimes c)=c \otimes a, \\
& \rho_{0}: A \otimes C \rightarrow A \otimes A, \quad \rho_{0}(a \otimes c)=a_{1} \otimes a_{2} \varepsilon_{C}(c) .
\end{aligned}
$$

Then it is easy to see that $A_{W_{0}, \rho_{0}} \otimes C$ is a crossed coproduct coalgebra, which is actually the tensor product coalgebra $A \otimes C$.

Assume that moreover $A_{W_{0}}^{\rho_{0}} \bowtie_{R}^{\sigma} C$ is a cross product bialgebra, that is the maps

$$
\begin{aligned}
& \Delta: A \otimes_{R, \sigma} C \rightarrow\left(A \otimes_{R, \sigma} C\right) \otimes\left(A \otimes_{R, \sigma} C\right), \quad \Delta(a \otimes c)=\left(a_{1} \otimes c_{1}\right) \otimes\left(a_{2} \otimes c_{2}\right), \\
& \varepsilon_{A} \otimes \varepsilon_{C}: A \otimes_{R, \sigma} C \rightarrow k,
\end{aligned}
$$

are algebra maps. Our aim is to describe all cross product bialgebras that are equivalent to $A_{W_{0}}^{\rho_{0}} \bowtie_{R}^{\sigma} C$.

Theorem 3.6 In the above hypotheses, a cross product bialgebra $A_{W^{\prime}}^{\rho^{\prime}} \bowtie_{R^{\prime}}^{\sigma^{\prime}} C$ is equivalent to $A_{W_{0}}^{\rho_{0}} \bowtie_{R}^{\sigma} C$ if and only if there exist linear maps $\theta, \gamma: C \rightarrow A \otimes C$, with notation $\theta(c)=$ $c_{<-1>} \otimes c_{<0>}$ and $\gamma(c)=c_{\{-1\}} \otimes c_{\{0\}}$, for all $c \in C$, such that the conditions (1.6)-(1.11) are satisfied and moreover we have, for all $a \in A, c \in C$ :

$$
\begin{aligned}
& W^{\prime}(a \otimes c)=\varepsilon_{A}\left(c_{<0>_{1-1\}}}\right) \varepsilon_{C}\left(c_{<0>_{\{0\}}}\right) c_{<0>_{10\}}} \otimes a c_{<-1>} c_{<0>_{2}\{-1\}}, \\
& \rho^{\prime}(a \otimes c)=\varepsilon_{C}\left(c_{<0>_{\{0\}}\{0\}}\right) a_{1} c_{<-1>_{1}} c_{<0>_{\{-1\}}} \otimes a_{2} c_{<-1>_{2}} c_{<0>_{\{0\}_{\{-1\}}}}, \\
& \varepsilon_{A}\left(c_{<-1>}\right) \varepsilon_{C}\left(c_{<0>}\right)=\varepsilon_{C}(c)=\varepsilon_{A}\left(c_{\{-1\}}\right) \varepsilon_{C}\left(c_{\{0\}}\right) \text {, } \\
& c_{<-1>} \otimes c_{<0>_{1}} \otimes c_{<0>_{2}}=c_{1_{<-1>}} \otimes c_{1_{<0>}} \otimes c_{2} \text {, } \\
& c_{\{-1\}} \otimes c_{\{0\}_{1}} \otimes c_{\{0\}_{2}}=c_{1_{\{-1\}}} \otimes c_{1_{\{0\}}} \otimes c_{2} \text {. }
\end{aligned}
$$

Proof. We prove first that if all those conditions are satisfied then $A_{W^{\prime}}^{\rho^{\prime}} \bowtie_{R^{\prime}}^{\sigma^{\prime}} C$ is indeed a cross product bialgebra and moreover it is equivalent to $A_{W_{0}}^{\rho_{0}} \bowtie_{R}^{\sigma} C$. We begin by obtaining some important consequences of the relations. We compute:

$$
\varepsilon_{C}\left(c_{1_{<0>}}\right) c_{1_{<-1>}} c_{2_{\{-1\}}} \otimes c_{2_{\{0\}}} \stackrel{3.10}{=} \varepsilon_{C}\left(c_{<0>1}\right) c_{<-1>} c_{<0>_{\{-1\}}} \otimes c_{<0>_{20\}}}
$$




$$
=c_{<-1>} c_{<0>_{\{-1\}}} \otimes c_{<0>_{\{0\}}}
$$

$\stackrel{1.9}{=} 1_{A} \otimes c$.

We apply $\varepsilon_{A} \otimes i d_{C}$ to this relation and we obtain:

$$
\begin{aligned}
c & =\varepsilon_{C}\left(c_{1_{<0>}}\right) \varepsilon_{A}\left(c_{1_{<-1>}}\right) \varepsilon_{A}\left(c_{2_{\{-1\}}}\right) c_{2_{\{0\}}} \\
& \stackrel{(3.9)}{=} \varepsilon_{C}\left(c_{1}\right) \varepsilon_{A}\left(c_{2_{\{-1\}}}\right) c_{2_{\{0\}}} \\
& =\varepsilon_{A}\left(c_{\{-1\}}\right) c_{\{0\}} .
\end{aligned}
$$

Also, for $c \in C$, we compute:

$$
\begin{aligned}
& \varepsilon_{C}\left(c_{1_{\{0\}}}\right) \varepsilon_{C}\left(c_{2_{\{0\}}}\right) c_{1_{\{-1\}}} \otimes c_{2_{\{-1\}}} \stackrel{3.11}{=} \varepsilon_{C}\left(c_{\{0\}_{1}}\right) \varepsilon_{C}\left(c_{\{0\}_{2}\{0\}}\right) c_{\{-1\}} \otimes c_{\{0\}_{2}\{-1\}} \\
& =\varepsilon_{C}\left(c_{\{0\}_{\{0\}}}\right) c_{\{-1\}} \otimes c_{\{0\}_{\{-1\}}} \text {. }
\end{aligned}
$$

Let us record for future use these three relations and the other three obtained by interchanging $<>$ and \{\} :

$$
\begin{aligned}
& \varepsilon_{C}\left(c_{1_{<0>}}\right) c_{1_{<-1>}} c_{2_{\{-1\}}} \otimes c_{2_{\{0\}}}=1_{A} \otimes c, \\
& \varepsilon_{C}\left(c_{1_{\{0\}}}\right) c_{1_{\{-1\}}} c_{2_{<-1>}} \otimes c_{2_{<0>}}=1_{A} \otimes c, \\
& \varepsilon_{A}\left(c_{<-1>}\right) c_{<0>}=c, \\
& \varepsilon_{A}\left(c_{\{-1\}}\right) c_{\{0\}}=c, \\
& \varepsilon_{C}\left(c_{1_{\{0\}}}\right) \varepsilon_{C}\left(c_{2_{\{0\}}}\right) c_{1_{\{-1\}}} \otimes c_{2_{\{-1\}}}=\varepsilon_{C}\left(c_{\{0\}_{\{0\}}}\right) c_{\{-1\}} \otimes c_{\{0\}_{\{-1\}}}, \\
& \varepsilon_{C}\left(c_{1_{<0>}}\right) \varepsilon_{C}\left(c_{2_{<0>}}\right) c_{1_{<-1>}} \otimes c_{2_{<-1>}}=\varepsilon_{C}\left(c_{<0><0>}\right) c_{<-1>} \otimes c_{<0><-1>},
\end{aligned}
$$

for all $c \in C$. Note that by using (3.15), the formula for $W^{\prime}$ may be written as

$$
W^{\prime}(a \otimes c)=\varepsilon_{C}\left(c_{<0>_{\{0\}}}\right) c_{<0>1} \otimes a c_{<-1>} c_{<0>2_{\{-1\}}} .
$$

We prove now that for $W^{\prime}$ and $\rho^{\prime}$ defined by (3.7) and respectively (3.8), $A_{W^{\prime}, \rho^{\prime}} \otimes C$ is a crossed coproduct coalgebra. The relations (3.1) and (3.2) for $W^{\prime}$ and $\rho^{\prime}$ are easy to prove and are left to the reader.

Proof of (3.3): We compute, for $a \in A$ and $c \in C$ :

$$
\begin{aligned}
& \left(\Delta_{C} \otimes i d_{A}\right)\left(W^{\prime}(a \otimes c)\right)=\varepsilon_{C}\left(c_{<0>_{\{0\}}}\right) c_{<0>1} \otimes c_{<0>2} \otimes a c_{<-1>} c_{<0>3_{\{-1\}}} \\
& \stackrel{3.10}{=} \varepsilon_{C}\left(c_{3_{\{0\}}}\right) c_{1_{<0>}} \otimes c_{2} \otimes a c_{1_{<-1>}} c_{3_{\{-1\}}}, \\
& \left(i d_{C} \otimes W^{\prime}\right) \circ\left(W^{\prime} \otimes i d_{C}\right) \circ\left(i d_{A} \otimes \Delta_{C}\right)(a \otimes c) \\
& =\left(i d_{C} \otimes W^{\prime}\right) \circ\left(W^{\prime} \otimes i d_{C}\right)\left(a \otimes c_{1} \otimes c_{2}\right) \\
& =\left(i d_{C} \otimes W^{\prime}\right)\left(\varepsilon_{C}\left(c_{1_{<0>2_{\{0\}}}}\right) c_{1_{<0>1}} \otimes a c_{1_{<-1>}>} c_{1_{<0>2_{\{-1\}}}} \otimes c_{2}\right) \\
& =\quad \varepsilon_{C}\left(c_{1_{<0>2}\{0\}}\right) c_{1_{<0>1}} \otimes \varepsilon_{C}\left(c_{2_{<0>2_{\{0\}}}}\right) c_{2_{<0>1}} \otimes a c_{1_{<-1>}} c_{1_{<0>2_{\{-1\}}}} c_{2_{<-1>}} c_{2_{<0>2_{\{-1\}}}} \\
& \stackrel{3.10}{=} \varepsilon_{C}\left(c_{1_{<0>2_{\{0\}}}}\right) c_{1_{<0>}} \otimes \varepsilon_{C}\left(c_{2_{\{0\}}}\right) c_{2_{1<0>}} \otimes a c_{1_{<-1>}>} c_{1_{<0>2_{\{-1\}}}} c_{2_{1_{<-1>}}} c_{2_{2_{\{-1\}}}}
\end{aligned}
$$


$=\quad \varepsilon_{C}\left(c_{1_{<0>2_{\{0\}}}}\right) c_{1_{<0>1}} \otimes \varepsilon_{C}\left(c_{3_{\{0\}}}\right) c_{2_{<0>}} \otimes a c_{1_{<-1>}} c_{1_{<0>2}\{-1\}} c_{2_{<-1>}} c_{3_{\{-1\}}}$

3.10

3.13

$\varepsilon_{C}\left(c_{2_{\{0\}}}\right) c_{1<0>} \otimes \varepsilon_{C}\left(c_{4_{\{0\}}}\right) c_{3<0>} \otimes a c_{1_{<-1>}} c_{2_{\{-1\}}} c_{3_{<-1>}>} c_{4_{\{-1\}}}$

$\varepsilon_{C}\left(c_{3\{0\}}\right) c_{1<0>} \otimes c_{2} \otimes a c_{1<-1>} c_{3_{\{-1\}}}, \quad$ q.e.d.

Proof of (3.4): We compute, for $a \in A$ and $c \in C$ :

$\left(\rho^{\prime} \otimes i d_{A}\right) \circ\left(i d_{A} \otimes W^{\prime}\right) \circ\left(\rho^{\prime} \otimes i d_{C}\right) \circ\left(i d_{A} \otimes \Delta_{C}\right)(a \otimes c)$

$=\left(\rho^{\prime} \otimes i d_{A}\right) \circ\left(i d_{A} \otimes W^{\prime}\right) \circ\left(\rho^{\prime} \otimes i d_{C}\right)\left(a \otimes c_{1} \otimes c_{2}\right)$

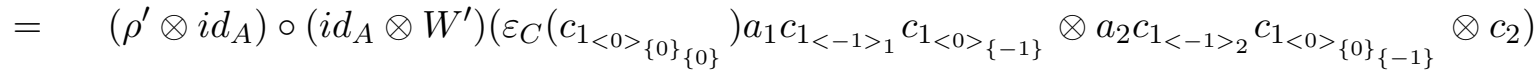

$=\left(\rho^{\prime} \otimes i d_{A}\right)\left(\varepsilon_{C}\left(c_{1_{<0>}\{0\}_{\{0\}}}\right) a_{1} c_{1_{<-1>1}} c_{1_{<0>\{-1\}}} \otimes \varepsilon_{C}\left(c_{2_{<0>2}\{0\}}\right) c_{2_{<0>1}}\right.$

$\left.\otimes a_{2} c_{1_{<-1>2}} c_{1_{<0>\{0\}}\{-1\}} c_{2_{<-1>}} c_{2_{<0>2}\{-1\}}\right)$

3.10

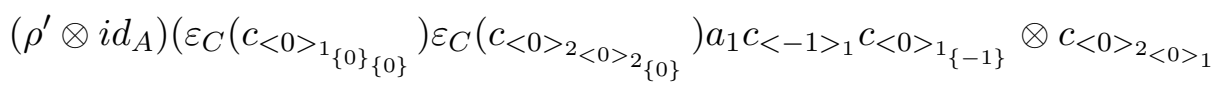

$\left.\otimes a_{2} c_{<-1>_{2}} c_{<0>_{1}\{\}_{\{-1\}}} c_{<0>_{2}<-1>} c_{<0>_{2}<0>_{2}\{-1\}}\right)$

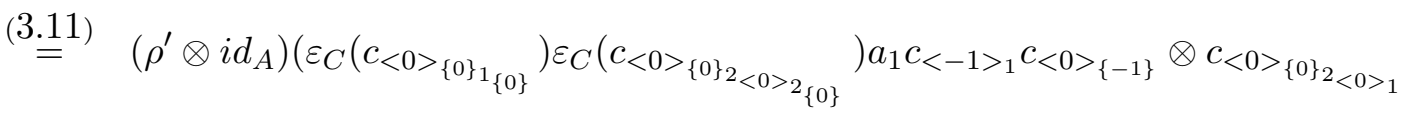

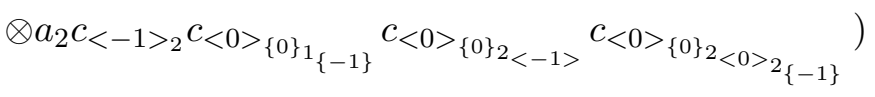

$\stackrel{3.13}{=}\left(\rho^{\prime} \otimes i d_{A}\right)\left(\varepsilon_{C}\left(c_{<0>_{\{0\}_{2}}\{0\}}\right) a_{1} c_{<-1>1} c_{<0>_{\{-1\}}} \otimes c_{<0>\{0\}_{1}} \otimes a_{2} c_{<-1>2} c_{<0>_{\{0\}_{2}}{ }_{\{-1\}}}\right)$

$\stackrel{3.11}{=}\left(\rho^{\prime} \otimes i d_{A}\right)\left(\varepsilon_{C}\left(c_{<0>2_{\{0\}}}\right) a_{1} c_{<-1>1} c_{<0>_{1-1\}}} \otimes c_{<0>1_{\{0\}}} \otimes a_{2} c_{<-1>_{2}} c_{<0>_{2}\{-1\}}\right)$

$\stackrel{3.10}{=}\left(\rho^{\prime} \otimes i d_{A}\right)\left(\varepsilon_{C}\left(c_{2_{\{0\}}}\right) a_{1} c_{1_{<-1>1}} c_{1_{<0>\{-1\}}} \otimes c_{1_{<0>\{0\}}} \otimes a_{2} c_{1_{<-1>2}} c_{2_{\{-1\}}}\right)$

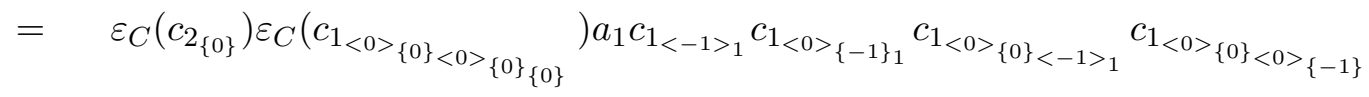

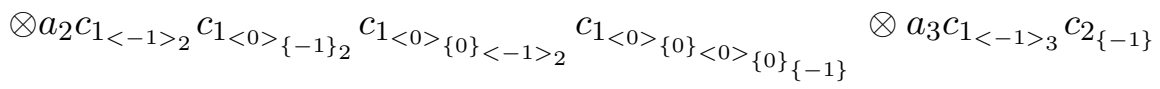

$=\varepsilon_{C}\left(c_{2_{\{0\}}}\right) \varepsilon_{C}\left(c_{1_{<0>}>_{\{0\}<0>\{0\}}}\right) a_{10\}} c_{1_{<-1>1}}\left(c_{1_{<0>}>_{\{-1\}}} c_{1_{<0>}>_{\{0\}}<-1>}\right) 1_{1} c_{1_{<0>}\{0\}<0>\{-1\}}$

$\otimes a_{2} c_{1_{<-1>2}}\left(c_{1_{<0>\{-1\}}} c_{\left.1_{<0>\{0\}<-1>}\right)}\right)_{2} c_{1_{<0>\{0\}<0>\{0\}_{\{-1\}}}} \otimes a_{3} c_{1_{<-1>3}} c_{2_{\{-1\}}}$

$\stackrel{1.10}{=} \varepsilon_{C}\left(c_{2_{\{0\}}}\right) \varepsilon_{C}\left(c_{1_{<0>}>_{\{0\}}\{0\}}\right) a_{1} c_{1_{<-1>1}} c_{1_{<0>}>_{-1\}}} \otimes a_{2} c_{1_{<-1>2}} c_{1_{<0>\{0\}}\{-1\}} \otimes a_{3} c_{1_{<-1>3}} c_{2_{\{-1\}}}$

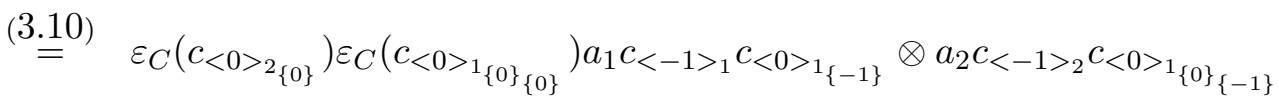

$\otimes a_{3} c_{<-1>_{3}} c_{<0>_{2}\{-1\}}$

$\stackrel{3.11}{=}$

$\varepsilon_{C}\left(c_{<0>}>_{\{0\}_{2}}\right) \varepsilon_{C}\left(c_{<0\}}>_{\{0\}_{\{0\}}}\right) a_{1} c_{<-1>1} c_{<0>_{\{-1\}}} \otimes a_{2} c_{<-1>2} c_{<0>_{\{0\}_{1}}}$

$\otimes a_{3} c_{<-1>3} c_{<0>}{ }_{\{0\}_{2}}{ }_{\{-1\}}$

3.11

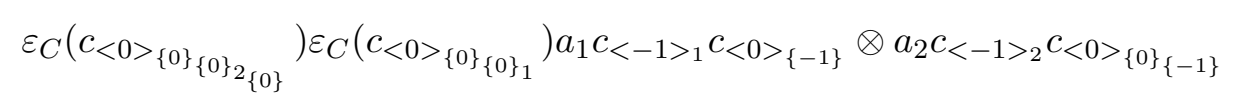




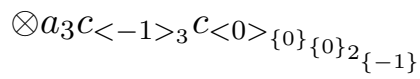

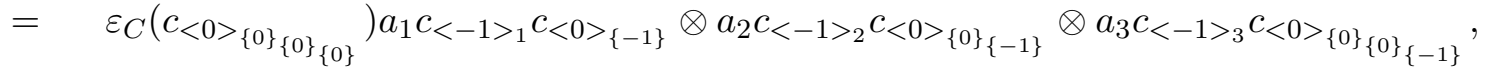

$$
\begin{aligned}
& \left(i d_{A} \otimes \rho^{\prime}\right) \circ\left(\rho^{\prime} \otimes i d_{C}\right) \circ\left(i d_{A} \otimes \Delta_{C}\right)(a \otimes c) \\
& =\left(i d_{A} \otimes \rho^{\prime}\right) \circ\left(\rho^{\prime} \otimes i d_{C}\right)\left(a \otimes c_{1} \otimes c_{2}\right) \\
& =\left(i d_{A} \otimes \rho^{\prime}\right)\left(\varepsilon_{C}\left(c_{1_{<0>\{0\}}\{0\}}\right) a_{1} c_{1_{<-1>1}} c_{1_{<0>}>_{-1\}}} \otimes a_{2} c_{1_{<-1>2}} c_{1_{<0>\{0\}_{\{-1\}}}} \otimes c_{2}\right) \\
& =\varepsilon_{C}\left(c_{1_{<0>\{0\}}\{0\}}\right) \varepsilon_{C}\left(c_{2_{<0>}\{0\}\{0\}}\right) a_{1} c_{1_{<-1>1}} c_{1_{<0>\{-1\}}} \\
& \otimes a_{2} c_{1<-1>2} c_{1<0>\{0\}_{\{-1\}_{1}}} c_{2_{<-1>1}} c_{2_{<0>}>_{\{-1\}}} \\
& \otimes a_{3} c_{1_{<-1>3}} c_{1_{<0>\{0\}_{\{-1\}_{2}}}} c_{2_{<-1>2}} c_{2_{<0>\{0\}_{\{-1\}}}} \\
& \stackrel{3.10}{=} \varepsilon_{C}\left(c_{<0>_{10\}_{\{0\}}}}\right) \varepsilon_{C}\left(c_{<0>_{2}<0>_{\{0\}}}\right) a_{10\}} c_{<-1>1} c_{<0>_{1-1\}}} \\
& \otimes a_{2} c_{<-1>_{2}}\left(c_{<0>1_{\{0\}_{\{-1\}}}} c_{<0>>_{2}<-1>}\right){ }_{1} c_{<0>_{2}<0>\{-1\}} \\
& \otimes a_{3} c_{<-1>3}\left(c_{<0>1_{\{0\}_{\{-1\}}}} c_{<0>_{2}<-1>}\right)_{2} c_{<0>_{2}<0>_{\{0\}_{\{-1\}}}} \\
& \stackrel{3.11}{=} \varepsilon_{C}\left(c_{<0>\{0\}_{10\}}}\right) \varepsilon_{C}\left(c_{<0>_{\{0\}_{2}<0>}{ }_{\{0\}}\{0\}}\right) a_{1} c_{<-1>1} c_{<0>\{-1\}} \\
& \otimes a_{2} c_{<-1>2}\left(c_{<0>_{\{0\}_{1-1\}}}} c_{<0>_{\{0\}_{2}}}\right){ }_{1} c_{<0>}>_{\{0\}_{2}<0>\{-1\}} \\
& \left.\otimes a_{3} c_{<-1>3}\left(c_{<0>_{\{0\}_{1}}{ }_{\{-1\}}} c_{<0>\{0\}_{2}<-1>}\right)\right)_{2} c_{<0>_{\{0\}_{2}}<0>_{\{0\}_{\{-1\}}}} \\
& \text { (3.13 } \\
& \varepsilon_{C}\left(c_{<0>\{0\}_{\{0\}}}\right) a_{1} c_{<-1>1} c_{<0>\{-1\}} \otimes a_{2} c_{<-1>2} c_{<0>\{0\}_{\{-1\}}} \\
& \otimes a_{3} c_{<-1>3} c_{<0>_{\{0\}_{\{0\}}}}, \text { q.e.d. }
\end{aligned}
$$

Proof of (3.5): Let $a \in A$ and $c \in C$; by using a part of the computation performed for proving (3.4), we compute:

$$
\begin{aligned}
& \left(W^{\prime} \otimes i d_{A}\right) \circ\left(i d_{A} \otimes W^{\prime}\right) \circ\left(\rho^{\prime} \otimes i d_{C}\right) \circ\left(i d_{A} \otimes \Delta_{C}\right)(a \otimes c) \\
& =\left(W^{\prime} \otimes i d_{A}\right)\left(\varepsilon_{C}\left(c_{2_{\{0\}}}\right) a_{1} c_{1_{<-1>1}} c_{1_{<0>\{-1\}}} \otimes c_{1_{<0>\{0\}}} \otimes a_{2} c_{1_{<-1>2}} c_{2_{\{-1\}}}\right)
\end{aligned}
$$

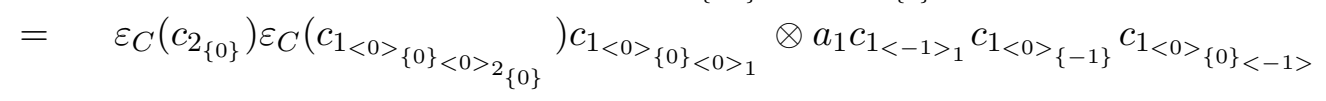

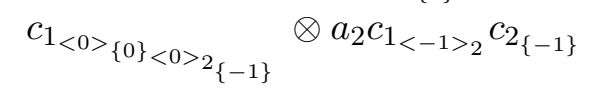

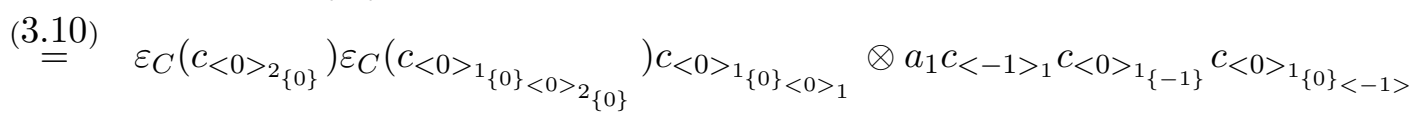

$$
\begin{aligned}
& c_{<0>1_{\{0\}}<0>2_{\{-1\}}} \otimes a_{2} c_{<-1>2} c_{<0>2_{\{-1\}}}
\end{aligned}
$$

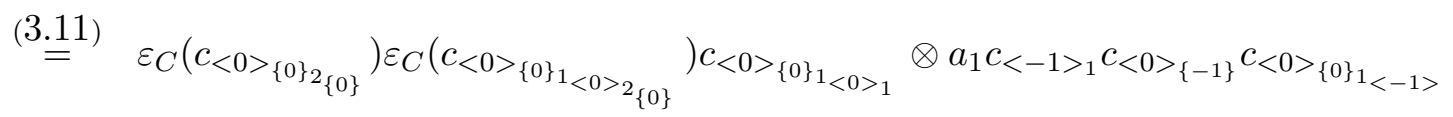

$$
\begin{aligned}
& c_{<0>_{\{0\}_{1<0>2}}} \otimes a_{2-1\}} c_{<-1>2} c_{<0>_{\{0\}_{2}}}
\end{aligned}
$$




$$
\begin{aligned}
& c_{<0>_{\{0\}_{1}}\{-1\}} \otimes a_{2} c_{<-1>2} c_{<0>}>_{\{0\}_{2}}{ }_{\{-1\}} \\
& =\varepsilon_{C}\left(c_{<0>_{\{0\}_{3}}}\right) \varepsilon_{C}\left(c_{<0>_{\{0\}_{2}}}\right) c_{<0\}} c_{\{0\}_{1}<0>} \otimes a_{1} c_{<-1>1} c_{<0>_{\{-1\}}} c_{<0>_{\{0\}_{1}}}{ }_{c_{<-1>}} \\
& c_{<0>_{\{0\}_{2}}} \otimes a_{2} c_{<-1\}}{ }_{2} c_{<0>_{\{0\}_{3}}} \\
& 3.10
\end{aligned}
$$

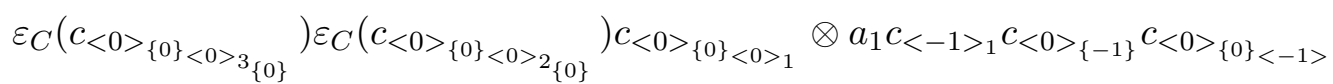

$$
\begin{aligned}
& c_{<0>}\{0\}_{<0>2_{\{-1\}}} \otimes a_{2} c_{<-1>2} c_{<0>}>_{\{0\}}<0>_{3-1\}} \\
& 1.10 \\
& \varepsilon_{C}\left(c_{<0>_{3}\{0\}}\right) \varepsilon_{C}\left(c_{<0>_{2}\{0\}}\right) c_{<0>_{1}} \otimes a_{1} c_{<-1>1} c_{<0>_{2}\{-1\}} \otimes a_{2} c_{<-1>_{2}} c_{<0>_{3-1\}}} \\
& \stackrel{3.100}{=} \varepsilon_{C}\left(c_{3_{\{0\}}}\right) \varepsilon_{C}\left(c_{2_{\{0\}}}\right) c_{1_{<0>}} \otimes a_{1} c_{1_{<-1>1}} c_{2_{\{-1\}}} \otimes a_{2} c_{1_{<-1>2}} c_{3_{\{-1\}}} \\
& 3.16 \\
& \varepsilon_{C}\left(c_{2_{\{0\}}}\right) c_{1_{<0>}} \otimes a_{1} c_{1_{<-1>1}} c_{2_{\{-1\}}} \otimes a_{2} c_{1_{<-1>2}} c_{2_{\{0\}}\{-1\}}, \\
& \left(i d_{C} \otimes \rho^{\prime}\right) \circ\left(W^{\prime} \otimes i d_{C}\right) \circ\left(i d_{A} \otimes \Delta_{C}\right)(a \otimes c) \\
& =\left(i d_{C} \otimes \rho^{\prime}\right) \circ\left(W^{\prime} \otimes i d_{C}\right)\left(a \otimes c_{1} \otimes c_{2}\right)
\end{aligned}
$$

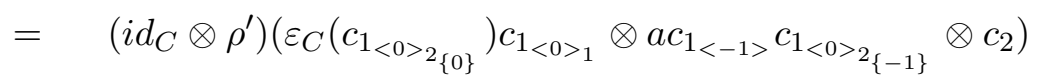

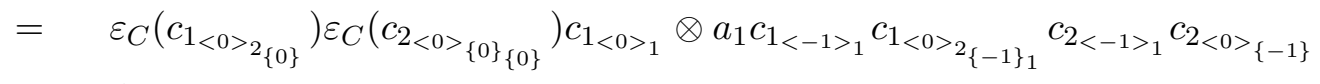

$$
\begin{aligned}
& \otimes a_{2} c_{1<-1>2} c_{1<0>_{2}\{-1\}_{2}} c_{2<-1>2} c_{2_{<0>\{0\}_{\{-1\}}}} \\
& \stackrel{3.10}{=} \varepsilon_{C}\left(c_{<0>_{1_{2}}\{0\}}\right) \varepsilon_{C}\left(c_{<0>_{2}<0>\{0\}}\right) c_{<0\}} c_{<01_{1}} \\
& \otimes a_{1} c_{<-1>1}\left(c_{\left.<0>_{1_{2}}, 1\right\}} c_{<0>_{2}<-1>}\right){ }_{1} c_{<0>_{2}<0>_{\{-1\}}} \\
& \otimes a_{2} c_{<-1>2}\left(c_{<0>_{2_{\{-1\}}}} c_{<0>_{2}<-1>}\right)_{2} c_{<0>_{2}<0>_{\{0\}}\{-1\}} \\
& =\varepsilon_{C}\left(c_{<0>_{2}\{0\}}\right) \varepsilon_{C}\left(c_{<0>_{3<0>}\{0\}_{\{0\}}}\right) c_{<0>1} \otimes a_{1} c_{<-1>1}\left(c_{<0>_{2}\{-1\}} c_{<0>_{3<-1>}}\right){ }_{1} c_{<0>_{3<0>}>_{\{-1\}}} \\
& \otimes a_{2} c_{<-1>_{2}}\left(c_{<0>_{2-1\}}} c_{<0>_{3}<-1>}\right)_{2} c_{<0>_{3}<0>_{\{0\}}\{-1\}} \\
& \stackrel{3.13}{=} \varepsilon_{C}\left(c_{<0>_{2}\{0\}_{\{0\}}}\right) c_{<0>1} \otimes a_{1} c_{<-1>1} c_{<0>_{2}\{-1\}} \otimes a_{2} c_{<-1>{ }_{2}} c_{<0>_{2}\{0\}_{\{-1\}}} \\
& \stackrel{3.10}{=} \varepsilon_{C}\left(c_{2_{\{0\}}\{0\}}\right) c_{1_{<0>}} \otimes a_{1} c_{1_{<-1>1}} c_{2_{\{-1\}}} \otimes a_{2} c_{1_{<-1>2}} c_{2_{\{0\}}\{-1\}} \text {, q.e.d. }
\end{aligned}
$$

So, $A_{W^{\prime}, \rho^{\prime}} \otimes C$ is indeed a crossed coproduct coalgebra. If we denote by $\Delta^{\prime}$ its comultiplication, then by $($ (3.6) $)$ we know that $\Delta^{\prime}$ is given by the formula $\Delta^{\prime}=\left(i d_{A} \otimes W^{\prime} \otimes i d_{C}\right) \circ\left(\rho^{\prime} \otimes \Delta_{C}\right) \circ\left(i d_{A} \otimes \Delta_{C}\right)$. We claim that this formula reduces to

$$
\Delta^{\prime}(a \otimes c)=a_{1} c_{1_{<-1>1}} c_{1_{<0>}\{-1\}} \otimes c_{1_{<0>}\{0\}} \otimes a_{2} c_{1_{<-1>2}} c_{2_{\{-1\}}} \otimes c_{2_{\{0\}}}
$$

for all $a \in A, c \in C$. Indeed, we compute:

$$
\begin{aligned}
\Delta^{\prime}(a \otimes c)= & \left(i d_{A} \otimes W^{\prime} \otimes i d_{C}\right) \circ\left(\rho^{\prime} \otimes \Delta_{C}\right)\left(a \otimes c_{1} \otimes c_{2}\right) \\
= & \left(i d_{A} \otimes W^{\prime} \otimes i d_{C}\right)\left(\varepsilon_{C}\left(c_{\left.1_{<0>\{0\}}\right)}\right) a_{1} c_{1_{<-1>1}} c_{1_{<0>\{-1\}}} \otimes a_{2} c_{1_{<-1>2}} c_{1_{<0>\{0\}}\{-1\}}\right. \\
& \left.\otimes c_{2} \otimes c_{3}\right)
\end{aligned}
$$




$$
\begin{aligned}
& =\quad \varepsilon_{C}\left(c_{\left.1_{<0>\{0\}}\right)}\right) a_{1} c_{1_{<-1>1}} c_{1_{<0>\{-1\}}} \otimes \varepsilon_{C}\left(c_{2_{<0>2_{\{0\}}}}\right) c_{2_{<0>1}} \\
& \otimes a_{2} c_{1_{<-1>2}} c_{1_{<0>\{0\}_{\{-1\}}}} c_{2_{<-1>}} c_{2_{<0>2}\{-1\}} \otimes c_{3} \\
& \stackrel{3.10}{=} \varepsilon_{C}\left(c_{1_{<0>}>_{\{0\}}\{0\}}\right) a_{1} c_{1_{<-1>}} c_{\left.1_{<0>}-1\right\}} \otimes \varepsilon_{C}\left(c_{2_{2}\{0\}}\right) c_{2_{1<0>}} \\
& \otimes a_{2} c_{1_{<-1>2}} c_{1_{<0>\{0\}_{\{-1\}}}} c_{2_{1<-1>}} c_{2_{2_{\{-1\}}}} \otimes c_{3}
\end{aligned}
$$

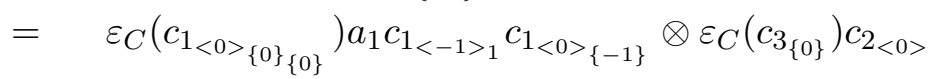

$$
\begin{aligned}
& \otimes a_{2} c_{1_{<-1>2}} c_{1_{<0>\{0\}_{\{-1\}}}} c_{2_{<-1>}} c_{3_{\{-1\}}} \otimes c_{4} \\
& \text { 3.11 } \varepsilon_{C}\left(c_{1_{<0>}>_{\{0\}_{\{0\}}}}\right) a_{1} c_{1_{<-1>1}} c_{1_{<0>_{\{-1\}}}} \otimes \varepsilon_{C}\left(c_{3_{\{0\}_{1}}}\right) c_{2_{<0>}} \\
& \otimes a_{2} c_{1_{<-1>2}} c_{1_{<0>\{0\}_{\{-1\}}}} c_{2_{<-1>}} c_{3_{\{-1\}}} \otimes c_{3_{\{0\}_{2}}} \\
& =\varepsilon_{C}\left(c_{1_{<0>}\{0\}}\right) a_{10\}} c_{1_{<-1>1}} c_{1_{<0>_{\{-1\}}}} \otimes c_{2_{<0>}} \\
& \otimes a_{2} c_{1_{<-1>2}} c_{1_{<0>\{0\}}{ }_{\{-1\}}} c_{2_{<-1>}} c_{3_{\{-1\}}} \otimes c_{3_{\{0\}}} \\
& \stackrel{3.16}{=} \varepsilon_{C}\left(c_{1_{<0>1_{\{0\}}}}\right) \varepsilon_{C}\left(c_{1_{<0>2}\{0\}}\right) a_{1} c_{1_{<-1>1}} c_{1_{<0>1_{\{-1\}}}} \otimes c_{2_{<0>}} \\
& \otimes a_{2} c_{1<-1>2} c_{1<0>2} c_{\{-1\}} c_{2<-1>} c_{3_{\{-1\}}} \otimes c_{3_{\{0\}}} \\
& \text { (3.10) } \varepsilon_{C}\left(c_{1_{1_{<0>}\{0\}}}\right) \varepsilon_{C}\left(c_{1_{2_{\{0\}}}}\right) a_{1} c_{1_{1_{<-1>1}}} c_{1_{\left.1_{<0>}-1\right\}}} \otimes c_{2_{<0>}} \\
& \otimes a_{2} c_{1_{1_{<-1>2}}} c_{1_{2_{\{-1\}}}} c_{2_{<-1>}} c_{3_{\{-1\}}} \otimes c_{3_{\{0\}}} \\
& =\varepsilon_{C}\left(c_{1<0>\{0\}}\right) \varepsilon_{C}\left(c_{2_{\{0\}}}\right) a_{1} c_{1_{<-1>1}} c_{1_{<0>}>_{\{-1\}}} \otimes c_{3<0>} \\
& \otimes a_{2} c_{1<-1>2} c_{2_{\{-1\}}} c_{3<-1>} c_{4_{\{-1\}}} \otimes c_{4_{\{0\}}} \\
& \stackrel{3.13}{=} \varepsilon_{C}\left(c_{1_{<0>}\{0\}}\right) a_{1} c_{1_{<-1>1}} c_{1_{<0>\{-1\}}} \otimes c_{2} \otimes a_{2} c_{1_{<-1>2}} c_{3_{\{-1\}}} \otimes c_{3_{\{0\}}} \\
& \stackrel{3.10}{=} \varepsilon_{C}\left(c_{<0>_{10\}}}\right) a_{1} c_{<-1>_{1}} c_{<0>_{1_{\{-1\}}}} \otimes c_{<0>_{2}} \otimes a_{2} c_{<-1>_{2}} c_{<0>_{3-1\}}} \otimes c_{<0>_{3}\{0\}} \\
& \stackrel{3.111}{=} \varepsilon_{C}\left(c_{<0>\{0\}_{1}}\right) a_{1} c_{<-1>1} c_{<0>_{\{-1\}}} \otimes c_{<0>_{\{0\}_{2}}} \otimes a_{2} c_{<-1>2} c_{<0>_{\{0\}_{3-1\}}}} \\
& \otimes c_{<0>}>_{\{0\}_{3}\{0\}} \\
& =\quad a_{1} c_{<-1>1} c_{<0>_{\{-1\}}} \otimes c_{<0>_{\{0\}_{1}}} \otimes a_{2} c_{<-1>_{2}} c_{<0>_{\{0\}_{2}}{ }_{\{-1\}}} \otimes c_{<0>_{\{0\}_{2}}{ }_{\{0\}}} \\
& \stackrel{3.11}{=} a_{1} c_{<-1>1} c_{<0>1_{\{-1\}}} \otimes c_{<0>1_{\{0\}}} \otimes a_{2} c_{<-1>2} c_{<0>2_{\{-1\}}} \otimes c_{<0>2_{\{0\}}} \\
& a_{1} c_{1_{<-1>1}} c_{1_{<0>}\{-1\}} \otimes c_{1_{<0>\{0\}}} \otimes a_{2} c_{1_{<-1>2}} c_{2_{\{-1\}}} \otimes c_{2_{\{0\}}}, \quad \text { q.e.d. }
\end{aligned}
$$

We have proved that $A_{W^{\prime}, \rho^{\prime}} \otimes C$ is a crossed coproduct coalgebra and we know from Theorem 1.2 that $A \otimes_{R^{\prime}, \sigma^{\prime}} C$ is a crossed product algebra. We have to prove now that $A_{W^{\prime}}^{\rho^{\prime}} \bowtie_{R^{\prime}}^{\sigma^{\prime}} C$ is a cross product bialgebra, that is the maps $\varepsilon_{A} \otimes \varepsilon_{C}: A \otimes_{R^{\prime}, \sigma^{\prime}} C \rightarrow k$ and $\Delta^{\prime}: A \otimes_{R^{\prime}, \sigma^{\prime}} C \rightarrow$ $\left(A \otimes_{R^{\prime}, \sigma^{\prime}} C\right) \otimes\left(A \otimes_{R^{\prime}, \sigma^{\prime}} C\right)$ are algebra maps. We will give an indirect proof. Define the map $\varphi: A_{W^{\prime}}^{\rho^{\prime}} \bowtie_{R^{\prime}}^{\sigma^{\prime}} C \rightarrow A_{W_{0}}^{\rho_{0}} \bowtie_{R}^{\sigma} C, \varphi(a \otimes c)=a c_{<-1>} \otimes c_{<0>}$. We know from Theorem 1.2 that $\varphi$ is an algebra isomorphism. We compute:

$$
\begin{aligned}
\left(\varepsilon_{A} \otimes \varepsilon_{C}\right) \circ \varphi(a \otimes c) & =\varepsilon_{A}\left(a c_{<-1>}\right) \varepsilon_{C}\left(c_{<0>}\right) \\
& =\varepsilon_{A}(a) \varepsilon_{A}\left(c_{<-1>}\right) \varepsilon_{C}\left(c_{<0>}\right)
\end{aligned}
$$




$$
\begin{array}{ll}
\stackrel{\sqrt[3.9]{=}}{=} & \varepsilon_{A}(a) \varepsilon_{C}(c) \\
= & \left(\varepsilon_{A} \otimes \varepsilon_{C}\right)(a \otimes c) .
\end{array}
$$

So $\left(\varepsilon_{A} \otimes \varepsilon_{C}\right) \circ \varphi=\varepsilon_{A} \otimes \varepsilon_{C}$, and since $\varepsilon_{A} \otimes \varepsilon_{C}: A \otimes_{R, \sigma} C \rightarrow k$ is an algebra map (because $A_{W_{0}}^{\rho_{0}} \bowtie_{R}^{\sigma} C$ is a cross product bialgebra) and $\varphi$ is an algebra isomorphism, it follows that $\varepsilon_{A} \otimes \varepsilon_{C}: A \otimes_{R^{\prime}, \sigma^{\prime}} C \rightarrow k$ is an algebra map. If we consider the comultiplication $\Delta$ of $A_{W_{0}}^{\rho_{0}} \bowtie_{R}^{\sigma} C$, which is defined by the formula $\Delta(a \otimes c)=a_{1} \otimes c_{1} \otimes a_{2} \otimes c_{2}$ and which is an algebra map from $A \otimes_{R, \sigma} C$ to $\left(A \otimes_{R, \sigma} C\right) \otimes\left(A \otimes_{R, \sigma} C\right)$ because $A_{W_{0}}^{\rho_{0}} \bowtie_{R}^{\sigma} C$ is a cross product bialgebra, it is very easy to see, by using the formula (3.19), that we have $\Delta^{\prime}=\left(\varphi^{-1} \otimes \varphi^{-1}\right) \circ \Delta \circ \varphi$, and since $\varphi$ is an algebra isomorphism it follows that $\Delta^{\prime}: A \otimes_{R^{\prime}, \sigma^{\prime}} C \rightarrow\left(A \otimes_{R^{\prime}, \sigma^{\prime}} C\right) \otimes\left(A \otimes_{R^{\prime}, \sigma^{\prime}} C\right)$ is an algebra map.

So, $A_{W^{\prime}}^{\rho^{\prime}} \bowtie_{R^{\prime}}^{\sigma^{\prime}} C$ is indeed a cross product bialgebra, and the relations $\left(\varepsilon_{A} \otimes \varepsilon_{C}\right) \circ \varphi=\varepsilon_{A} \otimes \varepsilon_{C}$ and $\Delta^{\prime}=\left(\varphi^{-1} \otimes \varphi^{-1}\right) \circ \Delta \circ \varphi$ show also that the map $\varphi$ is a coalgebra isomorphism, hence it is a bialgebra isomorphism. We know that $\varphi$ satisfies $\varphi\left(a \otimes 1_{C}\right)=a \otimes 1_{C}$, for all $a \in A$, hence $\varphi$ is a morphism of left $A$-modules, and the relation (3.10) implies that $\varphi$ is also a morphism of right $C$-comodules. Hence, the cross product bialgebras $A_{W^{\prime}}^{\rho^{\prime}} \bowtie_{R^{\prime}}^{\sigma^{\prime}} C$ and $A_{W_{0}}^{\rho_{0}} \bowtie_{R}^{\sigma} C$ are indeed equivalent.

Conversely, assume that $A_{W^{\prime}}^{\rho^{\prime}} \bowtie_{R^{\prime}}^{\sigma^{\prime}} C$ is a cross product bialgebra equivalent to $A_{W_{0}}^{\rho_{0}} \bowtie_{R}^{\sigma} C$, say via a map $\varphi: A_{W^{\prime}}^{\rho^{\prime}} \bowtie_{R^{\prime}}^{\sigma^{\prime}} C \simeq A_{W_{0}}^{\rho_{0}} \bowtie_{R}^{\sigma} C$. By Theorem 2.3, there exist linear maps $\theta, \gamma: C \rightarrow A \otimes C$, with notation $\theta(c)=c_{<-1>} \otimes c_{<0>}$ and $\gamma(c)=c_{\{-1\}} \otimes c_{\{0\}}$, for all $c \in C$, such that the conditions (1.6)-(1.11) are satisfied and moreover we have $\varphi(a \otimes c)=a c_{<-1>} \otimes c_{<0>}$ and $\varphi^{-1}(a \otimes c)=a c_{\{-1\}} \otimes c_{\{0\}}$ for all $a \in A$ and $c \in C$. By writing down the conditions that $\varphi$ and $\varphi^{-1}$ are morphisms of comodules, we obtain the conditions (3.10) and (3.11). By writing down the conditions that $\varphi$ and $\varphi^{-1}$ are counital, we obtain the condition (3.9). By writing down the condition that $\varphi$ is a coalgebra isomorphism, we obtain $\Delta^{\prime}=\left(\varphi^{-1} \otimes \varphi^{-1}\right) \circ \Delta \circ \varphi$, where $\Delta$ is the comultiplication of $A_{W_{0}}^{\rho_{0}} \bowtie_{R}^{\sigma} C$ and $\Delta^{\prime}$ is the comultiplication of $A_{W^{\prime}}^{\rho^{\prime}} \bowtie_{R^{\prime}}^{\sigma^{\prime}} C$, hence $\Delta^{\prime}$ is defined by the formula (3.19). Finally, from Remark 3.2, we can obtain the formulae for $W^{\prime}$ and $\rho^{\prime}$ by using the formula for $\Delta^{\prime}$, and an easy computation using the formulae (3.10) and (3.11) shows that $W^{\prime}$ and $\rho^{\prime}$ are given by the formulae (3.7) and respectively (3.8).

We present an example of equivalent cross product bialgebras, both of the type $A_{W}^{\rho} \bowtie_{R}^{\sigma} C$ where $A$ is a bialgebra and having the tensor product coalgebra structure. In [1, the authors introduced a construction, called unified product, which is a bialgebra of the type $A \ltimes H$, where $A$ is a bialgebra and $H$ is a coalgebra (with extra structures) and having the tensor product coalgebra structure. One can easily see that a unified product $A \ltimes H$ is a cross product bialgebra $A_{W_{0}}^{\rho_{0}} \bowtie_{R}^{\sigma} H$ (for certain $R$ and $\sigma$ ). If $A$ is a Hopf algebra and two unified products $A \ltimes H$ and $A \ltimes^{\prime} H$ are given such that they are both Hopf algebras, then [1], Theorem 3.4 provides a characterization of when $A \ltimes H$ and $A \ltimes^{\prime} H$ are (in our terminology) equivalent. If $A, A \ltimes H$ and $A \ltimes^{\prime} H$ are only bialgebras, Theorem 3.4 in [1] does not work anymore; but one can characterize when $A \ltimes H$ and $A \ltimes^{\prime} H$ are equivalent in this situation by using the above Theorem 3.6 .

There exists a "mirror version" of Brzeziński's crossed product (to be studied in detail elsewhere), as follows: if $\left(B, \mu, 1_{B}\right)$ is an (associative unital) algebra and $W$ is a vector space equipped with a distinguished element $1_{W} \in W$, then the vector space $W \otimes B$ is an associative algebra with unit $1_{W} \otimes 1_{B}$ and whose multiplication has the property that $(w \otimes b)\left(1_{W} \otimes b^{\prime}\right)=w \otimes b b^{\prime}$, for all $b, b^{\prime} \in B$ and $w \in W$, if and only if there exist linear maps $\nu: W \otimes W \rightarrow W \otimes B$ and 
$P: B \otimes W \rightarrow W \otimes B$ satisfying a certain list of conditions. The corresponding algebra structure is denoted by $W \bar{\otimes}_{P, \nu} B$. Similarly, there exist "mirror versions" for crossed coproduct coalgebras (of the type $D_{U, \eta} \bar{\otimes} Y$, where $D$ is a coalgebra and $Y$ is a vector space) and for cross product bialgebras, of the type $D_{U}^{\eta} \bar{P}_{P}^{\nu} B$. Such a cross product bialgebra becomes canonically a right $B$-module and a left $D$-comodule, we have an analogous concept of equivalence between two such cross product bialgebras and an analogue of Theorem 3.6 for them.

We present now an example of two equivalent cross product bialgebras in the above sense, of the type appearing in the "mirror version" of Theorem 3.6 (that is, one of them is of the form $D_{U}^{\eta}{ }_{P}^{\nu} B$, where $B$ is a bialgebra and the coalgebra structure is the tensor product coalgebra $D \otimes B)$. Namely, a well known result of Majid ([9]) asserts that the Drinfeld double of a finite dimensional quasitriangular Hopf algebra $H$ is isomorphic to a certain Radford biproduct. The explicit Radford biproduct that appears and the explicit isomorphism depend on the chosen explicit realization of the Drinfeld double of $H$. Unlike [9], we choose a realization of $D(H)$ on $H^{* c o p} \otimes H$, so the concrete formulae below are different from the ones in [9]; the same realization of the Drinfeld double was chosen in [8], where it was proved that, at the algebra level, the isomorphism in Majid's theorem is an example of invariance under twisting for twisted tensor products of algebras.

So, let $H$ be a finite dimensional Hopf algebra with antipode $S$. Recall that the Drinfeld double $D(H)$ is a Hopf algebra having $H^{* c o p} \otimes H$ as coalgebra structure and multiplication $(p \otimes h)\left(p^{\prime} \otimes h^{\prime}\right)=p\left(h_{1} \rightarrow p^{\prime} \leftarrow S^{-1}\left(h_{3}\right)\right) \otimes h_{2} h^{\prime}$, for all $p, p^{\prime} \in H^{*}$ and $h, h^{\prime} \in H$, where $\rightarrow$ and $\angle$ are the left and right regular actions of $H$ on $H^{*}$ given by $(h \rightarrow p)\left(h^{\prime}\right)=p\left(h^{\prime} h\right)$ and $(p \leftarrow h)\left(h^{\prime}\right)=p\left(h h^{\prime}\right)$.

Assume that $r=r^{1} \otimes r^{2} \in H \otimes H$ is a quasitriangular structure on $H$. Then on the vector space $H^{*}$ one can define certain structures that turn $H^{*}$ into a bialgebra in the Yetter-Drinfeld category ${ }_{H}^{H} \mathcal{Y} D$ (we denote it by $\underline{H}^{*}$ ) and we have a bialgebra isomorphism $\varphi: \underline{H}^{*} \times H \simeq D(H)$, $\varphi(p \otimes h)=p<S^{-1}\left(r^{1}\right) \otimes r^{2} h$, where $\underline{H}^{*} \times H$ is the Radford biproduct (cf. [11]).

One can see that both $\underline{H}^{*} \times H$ and $D(H)$ are "mirror versions" of cross product bialgebras between $H^{* c o p}$ and $H$, and for $D(H)=H^{* c o p} \otimes H$ we have that $H$ is a bialgebra and the coalgebra structure is the tensor product coalgebra. Obviously, we have $\varphi(\varepsilon \otimes h)=\varepsilon \otimes h$, for all $h \in H$, so $\varphi$ is a morphism of right $H$-modules, and one can easily check that $\varphi$ is also a morphism of left $H^{* c o p}$-comodules. Hence, $\underline{H}^{*} \times H$ and $D(H)$ are equivalent.

\section{References}

[1] A. L. Agore, G. Militaru, Extending structures II: The quantum version, J. Algebra 336 (2011), 321-341.

[2] A. L. Agore, G. Militaru, Deformations and descent type theory for Hopf algebras, arXiv:math.QA/1205.6564.

[3] Y. Bespalov, B. Drabant, Cross product bialgebras Part II, J. Algebra 240 (2001), 445-504.

[4] T. Brzeziński, Crossed products by a coalgebra, Comm. Algebra 25 (1997), 3551-3575.

[5] D. Bulacu, F. Panaite, F. Van Oystaeyen, Generalized diagonal crossed products and smash products for quasi-Hopf algebras. Applications, Comm. Math. Phys. 266 (2006), 355-399.

[6] A. Cap, H. Schichl, J. Vanzura, On twisted tensor products of algebras, Comm. Algebra 23 (1995), 4701-4735. 
[7] C. Di Luigi, J. A. Guccione, J. J. Guccione, Brzeziński's crossed products and braided Hopf crossed products, Comm. Algebra 32 (2004), 3563-3580.

[8] P. Jara Martínez, J. López Peña, F. Panaite, F. Van Oystaeyen, On iterated twisted tensor products of algebras, Internat. J. Math. 19 (2008), 1053-1101.

[9] S. Majid, Doubles of quasitriangular Hopf algebras, Comm. Algebra 19 (1991), 3061-3073.

[10] F. Panaite, Invariance under twisting for crossed products, Proc. Amer. Math. Soc. 140 (2012), 755-763.

[11] D. E. Radford, The structure of Hopf algebras with a projection, J. Algebra 92 (1985), $322-347$.

[12] A. Van Daele, S. Van Keer, The Yang-Baxter and Pentagon equation, Compositio Math. 91 (1994), 201-221. 